\title{
Manifestaciones menopaúsicas y calidad de vida en afrocolombianas. Valoración con escala Cervantes
}

\author{
Álvaro Monterrosa-Castro ${ }^{1}$, Leidy Carolina Durán-Méndez² ${ }^{2}$ Marlon Salguedo-Madrid³
}

\section{RESUMEN}

Introducción: las manifestaciones menopaúsicas se deben estudiar realizando consideraciones étnicas.

Objetivo: identificar las manifestaciones menopaúsicas más prevalentes y evaluar calidad de vida (CV) según estados menstruales.

Metodología: estudio que hace parte de CAVIMEC [Calidad de Vida en la Menopausia y Etnias Colombianas], realizado con Escala Cervantes que evalúa CV en la menopausia, en 646 afrocolombianas, entre 40-59 años, residentes en poblaciones del caribe y el pacífico.

Resultados: edad promedio 48,7 $\pm 5,7$ años, $69,1 \%$ con obesidad/sobrepeso, $22,7 \%$ con estudios tecnológicos/universitarios y 40,5\% posmenopáusicas. Las manifestaciones más prevalentes: oleadas de calor $76,4 \%$; sofocaciones $73,1 \%$; dolor músculo/articular $71,3 \%$; no conseguían dormir $61,8 \%$; sudoración sin esfuerzos 55,8\%; sequedad de piel 50,4\% y cefalea que aumentaba durante el día $44,2 \%$. La tercera parte no podían por los nervios, las cosas le aburrían, perdieron la capacidad de relajarse o notaban que todo le daba vueltas. El 24,4\% tenían menos interés en el sexo y 14,4\% tenían sexualidad nada importante. Las posmenopáusicas tuvieron peor puntuación en la mayoría de las manifestaciones y en los dominios salud, psíquico, sexualidad, relación de pareja, vasomotores, salud, envejecimiento y global.

Conclusión: oleadas de calor, sofocaciones y dolor musculo/articular fueron las manifestaciones más prevalentes, en siete de cada diez. Las posmenopáusicas tuvieron peor $C V$.

1 Médico. Especialista en ginecología y obstetricia. Profesor titular. Grupo de investigación salud de la mujer. Facultad de Medicina. Universidad de Cartagena. Colombia

2 Estudiante de pregrado. Grupo de Investigación Salud de la Mujer. Facultad de Medicina. Universidad de Cartagena. Colombia

3 Médico. Grupo de Investigación Salud de la Mujer. Facultad de Medicina. Universidad de Cartagena. Colombia

Correspondencia: Álvaro Monterrosa; alvaromonterrosa@gmail.com

Recibido: marzo 21 de 2017

Aceptado: abril 25 de 2017

Cómo citar: Monterrosa-Castro A, Durán-Méndez LC, Salguedo-Madrid M. Manifestaciones menopaúsicas y calidad de vida en afrocolombianas. Valoración con escala Cervantes. latreia. 2017 0ct-Dic;30(4): 376-390. DOI 10.17533/udea.iatreia.v30n4a03. 


\section{PALABRAS CLAVE}

Calidad de Vida; Climaterio; Menopausia

\section{SUMMARY}

Menopausal manifestations and quality of life in afro-colombians. Valuation whit Cervantes scale

Introduction: the menopausal manifestations should be studied by ethnic considerations.

Objective: to identify the most prevalent menopausal manifestations and to evaluate quality of life according to menstrual states.

Methods: this study is a part of CAVIMEC [Quality of Life in Menopause and Colombian Ethnic Groups], performed with Cervantes Scale, which evaluates $C V$ in menopause, in 646 Afro-Colombians, aged 40-59 years, living in populations of the Caribbean and Pacific.

Results: mean age $48.7 \pm 5.7$ years, $69.1 \%$ with obesity/ overweight, $22.7 \%$ with university/technological studies and $40.5 \%$ postmenopausal. The most prevalent manifestations: hot flashes $76.4 \%$; suffocation $73.1 \%$; Muscle/joint pain $71.3 \%$; they couldn't sleep $61.8 \%$; easy sweating episode 55.8\%; dry skin $50.4 \%$ and headache that increased during the day $44.2 \%$. The third part could not be good by the nerves, things like boring, lost the ability to relax or noticed that everyone was spinning $24.4 \%$ had less interest in sex and $14.4 \%$ had not significant sexuality. Postmenopausal women had worse scores in most of the manifestations and in the domains of health, psychic, sexuality, relationship, vasomotor, health, aging and global.

Conclusion: hot flashes, suffocations and muscle/ joint pain were the manifestations most prevalent, in seven out of ten. Postmenopausal women had worse quality of life.

\section{KEYWORDS}

Climacteric; Menopause; Quality of Life

\section{RESUMO}

Manifestações menopáusicas e qualidade de vida em afro-colombianas. Valorização com escala Cervantes

Introdução: as manifestações menopáusicas se devem estudar realizando considerações étnicas.
Objetivo: identificar as manifestações menopáusicas mais prevalentes e avaliar qualidade de vida (QV) segundo estados menstruais.

Metodologia: estudo que faz parte de CAVIMEC [Qualidade de Vida na Menopausa e Etnias Colombianas], realizado com Escala Cervantes que avalia QV na menopausa, em 646 afro-colombianas, entre 40-59 anos, residentes em populações do caribe e do pacífico.

Resultados: Idade média 48,7 $\pm 5,7$ anos, $69,1 \%$ com obesidade/sobrepeso, 22,7\% com estudos tecnológico-universitários e 40,5\% pós-menopáusicas. As manifestações mais prevalentes: ondas de calor $76,4 \%$; sufocações $73,1 \%$; dor muscular/articular $71,3 \%$; não conseguem dormir $61,8 \%$; suar sem esforços $55,8 \%$; Pele seca $50,4 \%$ e cefaleia que aumentava durante o dia $44,2 \%$. A terceira parte não podia por medo, as coisas the aborreciam, perderam a capacidade de relaxar-se ou notavam que tudo girava na sua $v$ olta. $24,4 \%$ tinham menos interesse pelo sexo e $14,4 \%$ tinham a sexualidade sem importância. As pós-menopáusicas tiveram pior pontuação na maioria das manifestações e nos domínios saúde, psíquico, sexualidade, relação do casal, vasomotores, saúde, envelhecimento e global.

Conclusão: ondas de calor, sufocações e dor muscular/ articular foram as manifestações mais prevalentes, em sete de cada dez. As pós-menopáusicas tiveram pior $C V$.

\section{PALAVRAS CHAVE}

Qualidade de Vida; Climatério; Menopausa.

\section{INTRODUCCIÓN}

Lacalidad de vida(CV)esun concepto multidimensional que ha sido abordado en diferentes campos del conocimiento, especialmente desde la sociología, política, marketing, condiciones ambientales o climáticas, así como desde la salud (1). En esta área, una de las estrategias para establecer el deterioro de la CV es la aplicación de escalas que permitan valorar la presencia y severidad de diversas manifestaciones que modifican las condiciones de bienestar. Aunque las escalas de $C \nabla$ son subjetivas, permiten identificar desde la percepción del individuo manifestaciones 
biológicas, psicológicas o sociales, las cuales pueden ser interpretadas como indicadores de satisfacción, felicidad, expectativas o deterioro de la salud (2-5).

La menopausia es el instante biológico del cese definitivo de la menstruación y hace parte del climaterio, etapa del ciclo vital de la mujer, que sigue a la reproductiva, sin extremos bien definidos aunque en general se consideran los 40-59 años. Se han señalado diversas manifestaciones menopáusicas: oleadas de calor, dolor músculo/articular, trastornos del sueño, alteraciones en el estado de ánimo, irritabilidad o ansiedad, problemas sexuales, disfunción vesical y sequedad vaginal, entre otras (6-8).

Las manifestaciones relacionadas con el climaterio varían entre grupos $(7,9)$. Las mujeres de raza negra tienen mayor presencia de manifestaciones vasomotoras que las caucásicas, tanto premenopáusicas como posmenopáusicas $(6,10,11)$. En "Study of Woman`s Health Across The Nation" [SWAN], valoración transversal realizada en 16065 mujeres con 40-55 años de edad, se señaló que en Estados Unidos existen diferencias en la frecuencia de oleadas de calor, según grupos raciales, siendo en afroamericanas $45,6 \%$, hispánicas 33,5\%, caucásicas 31,2\%, chinas 20,5\% y Japonesas $17,6 \%$ (12).

Existe la necesidad que en comunidades afrocolombianas se identifiquen las manifestaciones menopáusicas que más se presentan, para a partir de los hallazgos se diseñen políticas sanitarias ajustadas a dicha condición étnica. El objetivo fue identificar las manifestaciones menopaúsicas más prevalentes y evaluar la $C V$ según estados menstruales, en afrocolombianas.

\section{METODOLOGÍA}

CAVIMEC [Calidad de Vida en la Menopausia y Etnias Colombianas] es un proyecto de investigación transversal iniciado en el año 2008 y proyectado al 2025 , que involucra mujeres colombianas saludables entre 40-59 años de edad, que realizan actividades cotidianas, residentes en áreas urbanas o rurales, de distintas etnias y captadas en sus viviendas por encuestadoras, quienes explican los alcances de la investigación y las motivan para participar en el llenado anónimo de los formatos. Se solicitan datos personales, sociodemográficos (paridad, estado menstrual, uso actual de la terapia hormonal, nivel educativo, estado civil y nutricional, actividad laboral, hábito de fumar) y se aplican escalas para valorar $C \nabla$. Se excluyen mujeres con desórdenes mentales y discapacidad para llenar los documentos, analfabetas, las que no comprenden las preguntas de los formularios, las que se desean retirar después de iniciar la participación y las embarazadas. Los Formularios incompletos son eliminados. Los correctamente diligenciados son digitados en la base de datos CAVIMEC. EI presente estudio es un análisis parcial de datos de dicha base.

Participantes: mujeres afrocolombianas que cumplieron los siguientes criterios: $[\mathrm{A}]$ en la pregunta "etnia a la cual pertenece" del formulario de datos sociodemográficos, informaron ser afrodescendiente, mulata, negra, palenquera o raizal. [B] tener rasgos fenotípicos de raza negra. [C] ser hijas de padre y madre de raza negra. [D] Residir en áreas rurales o barrios considerados asentamientos afrodescendientes. Las estudiadas fueron tomadas de Barranquilla y Cartagena (región caribe), Cali (costa pacífica), Buenarentura (departamento del Valle) y San Basilio de Palenque, San Cayetano, Mahates, San Pablo y Marialabaja (departamento de Bolívar).

Tamaño de muestra: el último censo de población de Colombia fue realizado en el año 2005, se contaron 21.718.757 mujeres, estando 4.423.739 entre 40-59 años de edad. Además se señaló que el $10,62 \%$ se auto reconocieron como negro, mulato, afrocolombiano, raizal o palenquero. La proyección al año 2016 estimó 24.678 .673 de mujeres, estando 5.660 .856 en ese mismo rango de edad (13), por tanto se consideran 601.182 mujeres afrocolombianas de 40-59 años de edad como el universo del estudio y se calculó tamaño de muestra de 633 mujeres con nivel de heterogeneidad del 50\%, margen de error del $5 \%$ y nivel de confianza del $99 \%$.

Definiciones: el estado nutricional se estableció utilizando la propuesta de OMS de acuerdo aI IMC, categorizándose como: infrapeso, normal, sobrepeso y obesidad. El nivel de escolaridad fue clasificado según número de años de estudios: primarios, secundarios, técnico, tecnólogo y profesional. Además se subdividieron los años de estudio en doce o más años y menos de doce. Se consideraron los estados civiles y la actividad laboral dentro o fuera de casa. Fueron agrupadas en premenopáusicas, las que 
presentaban períodos menstruales o tenían menos de un año de amenorrea y posmenopáusicas, las que tenían uno o más años sin episodios menstruales. Las últimas fueron subdivididas en tempranas, tardía y alejada.

Escala Cervantes (EC): cuestionario validado que mide desde la percepción de la mujer, 31 manifestaciones o condiciones de salud en la menopausia, en las cuatro semanas anteriores. Las preguntas se agrupan en 4 dominios: Menopausia y salud, que incluye los subdominios: síntomas vasomotores, salud y envejecimiento. Psíquico. Sexualidad. Relación de pareja. Cada pregunta es una manifestación de salud, que se responde tipo Likert: 0 (no presente), 1-2-3-4 (estados intermedios) y 5 (mucho), siendo de mejor a peor en las preguntas negativas $[1,2,3,5,6,7,9,10,11,12,14$, $16,17,18,19,21,23,24,25,27,28,29,31]$ y lo contrario en las positivas $[4,8,13,15,20,22,26,30]$. Las sumas crean el puntaje de los dominios y entre estos el global, que va de 0-155. A mayor puntuación, mayor deterioro de los dominios y de la $C \nabla$. No se ha propuesto punto de corte de la EC para establecer deterioro de la CV (5).

Aspectos éticos: todas las participantes firmaron consentimiento informado previo a la aplicación de la encuesta, de acuerdo a la declaración de Helsinki. Se tuvieron en cuenta normas científicas, técnicas y administrativas para la investigación en salud, establecidas en la resolución 8430 de 1993 del Ministerio de Salud de la República de Colombia, lo que permite considerar al estudio como investigación sin riesgos (14). Ninguna residía en resguardos étnicos. CAVIMEC está aprobado por el comité de ética de la Universidad de Cartagena.

Análisis estadístico: realizado con EPI-INFO-7(Centers for Disease Control and Prevention, Atlanta, EEUU; 2008). Los datos se expresan en medias con desviación estándar para datos continuos y en valores absolutos, porcentajes e intervalos de confianza los categóricos. Las diferencias fueron evaluadas con ANOVA o Test de Student y Mann-Witney o Kruskal-Wallis, según homogeneidad de la varianza. Los porcentajes fueron evaluados usando el $\chi 2$. Un valor de $\mathrm{P}<0.05$ fue considerado estadísticamente siognificativo. Se realizó regresión lineal simple no ajustada para establecer los factores de riesogo asociados a puntuación global de la EC por encima de la media.

\section{RESULTADOS}

La base de datos CAVIMEC está constituida por información aportada por 4780 colombianas, de diferentes regiones $y$ etnias, con edades entre 40-59 años. A 1895 (39.6\%) se les ha aplicado la EC y de ellas $646(34,1 \%)$ se clasificaron como afrocolombianas.

En la tabla 1 las características sociodemográficas. Edad 48,7 $\pm 5,7$ años, 3,5 $\pm 2,1$ hijos y el $67,6 \%$ actualmente unidas. IMC $27,2 \pm 4,8$, estando el $69,1 \%$ en obesidad o sobrepeso. Nivel de escolaridad 8,0 $\pm 5,7$ años, teniendo el $42,2 \%$ nivel primario mientras que el 22,7\% habían realizado estudios tecnológicos o profesionales. La cuarta parte tenía doce o más años de escolaridad. El 48,0\% cumplían labores de ama de hogar $y$ el 26,7\% comerciantes. El 9,4\% declararon ser fumadoras y el 4,8\% utilizaban terapia hormonal. EI $40,5 \%$ en posmenopausia, siendo edad de última menstruación $47,1 \pm 4,0$, la mitad en posmenopausia temprana y el número de años desde la última menstruación fue 6,2 $\pm 4,4$.

EI alpha de Cronbach encontrado para EC: 0,819. Las manifestaciones que hacen referencia a las oleadas de calor fueron las más frecuentes: 76,4\% informó oleadas de calor de repente, $73,1 \%$ notaban sofocaciones $y$ $55,8 \%$ sudoración sin hacer esfuerzos. El $71,3 \%$ se quejaban de dolor músculo/articular, mientras que $43,9 \%$ de hormigueo en manos/pies. El 50,4\% notó mayor sequedad de piel, $61,8 \%$ no conseguían dormir suficientemente, $56,1 \%$ dormían pero no descansaban y $49,7 \%$ desde que se levantaban se encontraban cansadas. El 44,2\% manifestó que durante el día se les incrementaba el dolor de cabeza $y$ un porcentaje similar consideró que las alteraciones de salud le afectaban el trabajo doméstico. El 13,3\% sentía que no tenía buena salud como otras personas de su edad.

La tercera parte informó que no podían más por el estado de nervios o que las cosas le aburrían. Igual proporción manifestó haber perdido la capacidad de relajarse o notaba que las cosas le daban vueltas. El $28 \%$ se sentían hinchadas creyendo que retenían líquidos, el $20 \%$ se sentían vacías, el 28\% notaban que el corazón les latía de prisa sin control y el 25\% sentían picor a consecuencia de la sequedad vaginal. A dos de cada diez le daba miedo presentar salida espontánea o incontrolada de orina, mientras que el $15 \%$ informó sentir que no servía para nada, que no 
Tabla 1. Características sociodemográficas n=646

\begin{tabular}{|c|c|}
\hline Edad, $\mathrm{X} \pm \mathrm{SD}$ & $48,7 \pm 5,7$ \\
\hline $\mathrm{IMC}, \mathrm{X} \pm \mathrm{SD}$ & $27,2 \pm 4,8$ \\
\hline Hijos, $\mathrm{X} \pm \mathrm{SD}$ & $3,5 \pm 2,1$ \\
\hline Estudio, $\mathrm{X} \pm \mathrm{SD}$ & $8,0 \pm 5,7$ \\
\hline \multicolumn{2}{|c|}{ Grupos etarios, n (\%) [IC95\%] } \\
\hline $40-44$ & $165(25,5)[22,2-29,1]$ \\
\hline $45-49$ & $205(31,7)[28,1-35,5]$ \\
\hline $50-54$ & $136(21,0)[18,0-24,4]$ \\
\hline $55-59$ & $140(21,6)[18,5-25,0]$ \\
\hline \multicolumn{2}{|c|}{ Estados nutricionales, n (\%) [IC95\%] } \\
\hline Infrapeso $\left(<18,50 \mathrm{~kg} / \mathrm{m}^{2}\right)$ & $15(2,3)[1,3-3,8]$ \\
\hline Normal $\left(18,50-24,99 \mathrm{~kg} / \mathrm{m}^{2}\right)$ & $184(28,4)[25,0-32,1]$ \\
\hline Sobrepeso $\left(25,00-29,99 \mathrm{~kg} / \mathrm{m}^{2}\right)$ & $285(44,1)[40,2-48,0]$ \\
\hline Obesidad I ( $\left.30,00-34,99 \mathrm{~kg} / \mathrm{m}^{2}\right)$ & $112(17,3)[14,5-20,5]$ \\
\hline Obesidad II $(35,00$ - 39,99 kg/m²) & $42(6,5)[4,7-8,7]$ \\
\hline Obesidad III $\left(\geq 40,00 \mathrm{~kg} / \mathrm{m}^{2}\right)$ & $8(1,2)[0,5-2,5]$ \\
\hline \multicolumn{2}{|c|}{ Nivel de escolaridad, n (\%) [IC95\%] } \\
\hline Primario (0-5 años de estudio) & $273(42,2)[38,4-46,1]$ \\
\hline Bachillerato (6-12 años de estudio) & $208(32,2)[28,6-35,9]$ \\
\hline Técnico (13-14 años de estudio) & $18(2,7)[1,7-4,4]$ \\
\hline Tecnólogo (15-16 años de estudio) & $13(2,0)[1,1-3,5]$ \\
\hline Profesional (17 y más años de estudio) & $134(20,7)[17,7-24,1]$ \\
\hline \multicolumn{2}{|c|}{ Años de formación académica, n (\%) [IC95\%] } \\
\hline Menos de doce años & $481(74,4)[63,2-68,5]$ \\
\hline Doce y más años & $165(25,5)[31,4-36,7]$ \\
\hline \multicolumn{2}{|c|}{ Actividad laboral, n (\%) [IC95\%] } \\
\hline Ama de Hogar & $311(48,1)[44,2-52,0]$ \\
\hline Oficina & $28(4,3)[2,9-6,2]$ \\
\hline Comerciante & $173(26,7)[23,4-30,4]$ \\
\hline Obrera & $2(0,3)[0,0-1,2]$ \\
\hline Jubilada & $9(1,3)[0,6-2,7]$ \\
\hline Profesional & $123(19,0)[16,1-22,3]$ \\
\hline \multicolumn{2}{|c|}{ Realización de la actividad laboral, n (\%) [IC95\%] } \\
\hline En casa & $320(49,5)[45,6-53,4]$ \\
\hline Fuera de casa & $326(50,4)[46,5-54,3]$ \\
\hline \multicolumn{2}{|c|}{ Estado civil, n (\%) [IC95\%] } \\
\hline Soltera & $84(13,0)[10,5-15,9]$ \\
\hline Casada & $232(35,9)[32,2-39,7]$ \\
\hline Unión libre & $205(31,7)[28,1-35,5]$ \\
\hline Separada & $60(9,2)[7,2-11,8]$ \\
\hline Viuda & $65(10,0)[7,9-12,7]$ \\
\hline \multicolumn{2}{|c|}{ Habido de fumar, n (\%) [IC95\%] } \\
\hline Nunca & $516(79,8)[76,5-82,8]$ \\
\hline Anteriormente & $69(10,6)[8,4-13,3]$ \\
\hline Actualmente & $61(9,4)[7,3-12,0]$ \\
\hline
\end{tabular}

CONTINÚA 
Tabla 1. Características sociodemográficas $n=646$ (Continuación)

\begin{tabular}{|c|c|}
\hline \multicolumn{2}{|c|}{ Uso actual de terapia hormonal, n (\%) [IC95\%] } \\
\hline No & $615(95,2)[93,1-96,6]$ \\
\hline Sí & $31(4,8)[3,4-6,8]$ \\
\hline \multicolumn{2}{|c|}{ Estado menstrual, n (\%) [IC95\%] } \\
\hline Premenopausia & $384(59,5)[55,1-63,8]$ \\
\hline Posmenopausia & $262(40,5)[36,7-44,4]$ \\
\hline \multicolumn{2}{|c|}{ Posmenopáusicas, $n=262, X \pm S D$} \\
\hline Edad de última menstruación & $47,1 \pm 4,0$ \\
\hline Años desde la última regla & $6,2 \pm 4,4$ \\
\hline \multicolumn{2}{|c|}{ Edad de establecimiento de la menopausia, n (\%) [IC95\%] } \\
\hline Antes de los 40 años & $6(2,2)[0,8-4,9]$ \\
\hline Entre $40-44$ años & $51(19,4)[14,8-24,7]$ \\
\hline Entre $45-49$ años & $129(49,2)[43,0-55,4]$ \\
\hline 50 o más años & $76(29,0)[23,5-34,9]$ \\
\hline \multicolumn{2}{|c|}{ Clasificación de las etapas en la postmenopausia, n (\%) [IC95\%] } \\
\hline Posmenopausia temprana (0-5años) & $133(50,7)[44,5-56,9]$ \\
\hline Posmenopausia tardía (6-10 años) & $91(34,7)[28,9-40,8]$ \\
\hline Posmenopausia alejada (+10 años) & $38(14,5)[10,4-19,3]$ \\
\hline
\end{tabular}

le importaría estar muerta o creían que los demás estarían mejor si ella.

El 41,8\% manifestó tener notoriamente menos relaciones sexuales que antes, el $27,8 \%$ no estaban satisfechas con sus relaciones sexuales, el $24,4 \%$ tenían menos interés en el sexo que antes y 14,4\% informaron tener vida sexual nada importante. EI 29,5\% consideró que nunca eran tratadas de igual a igual en su relación de pareja, el 16,5\% no eran para nada feliz con su relación de pareja y el 13,4\% sentenciaron que su papel como esposa era nada importante. Tabla 2.

Las mujeres fueron agrupadas según estados menstruales, tabla 3 presenta puntuación media con desviación estándar de cada manifestación. No se observó diferencia significativa en: "estar satisfecha con las relaciones sexuales", "sentirse tratada de igual a igual en la relación de pareja” y "notar sofocaciones". Las preguntas: "el interés por el sexo se mantiene como siempre", "considerarse feliz en la relación de pareja”, "tener relaciones sexuales tan a menudo como antes" y "en la vida el sexo es extremadamente importante", tuvieron peor evaluación en premenopáusicas $(p<0,005)$. A su vez las posmenopáusicas tuvieron peor evaluación en las otras 24 preguntas, con diferencias significativas, siendo las de peor puntuación: "notar mucho calor de repente" $y$ "notar que las articulaciones duelen". La puntuación para toda la población fue: 39,2 $\pm 22,6$, siendo peor en posmenopáusicas $(p<0,001)$, quienes tuvieron peor puntuación en todos los dominios $y$ subdominios que las premenopáusicas $(\mathrm{p}<0,001)$. Tabla 4. De las estudiadas, 257 (39,7\%) tuvieron puntuación por encima de la media.

Mayor rango de edad fue factor asociado no ajustado a puntuación por encima de la media de EC, siendo significativo en todos los grupos etarios, OR: 11,3 [IC95\%:6,8-18,6] para 55-59 años. Igual comportamiento tuvo infrapeso, sobrepeso, obesidad en diferentes categorías, menos de 12 años de educación, fumar anteriormente y en la actualidad, ausencia de uso de terapia hormonal $y$ posmenopausia $(\mathrm{p}<0,001)$. La postmenopausia tardía y alejada, fueron factores asociados respectivamente a dos y cinco veces puntuación por encima de la media, que postmenopausia temprana. Tabla 5. 
Tabla 2. Manifestaciones menopáusicas identificadas con escala cervantes. Presencia y severidad $\mathrm{n}=646$

\begin{tabular}{|c|c|c|c|c|}
\hline & ÍTEM & NINGÚN PUNTO & $\begin{array}{r}\text { 1-4 PUNTOS } \\
\text { n (\%) [IC95\%] }\end{array}$ & 5 PUNTOS \\
\hline \multirow{2}{*}{1} & \multirow{2}{*}{$\begin{array}{l}\text { Durante el día noto que la cabeza me } \\
\text { va doliendo cada vez más }\end{array}$} & Nunca & Intermedio & Todos los días \\
\hline & & $361(55,8)[51,9-59,7]$ & $272(42,1)[38,2-46,0]$ & $13(2,01)[1,1-3,5]$ \\
\hline \multirow{2}{*}{2} & \multirow{2}{*}{$\begin{array}{l}\text { No puedo más } \\
\text { de lo nerviosa que estoy }\end{array}$} & Nunca & Intermedio & Constantemente \\
\hline & & $446(69,0)[65,2-72,5]$ & $191(29,5)[26,1-33,2]$ & $9(1,3)[0,6-2,7]$ \\
\hline \multirow{2}{*}{3} & \multirow{2}{*}{ Noto mucho calor de repente } & Nunca & Intermedio & Constantemente \\
\hline & & $152(23,5)[20,3-27,0]$ & $364(56,3)[52,4-60,2]$ & $130(20,1)[17,1-23,4]$ \\
\hline \multirow{2}{*}{4} & \multirow{2}{*}{$\begin{array}{l}\text { Mi interés por el sexo } \\
\text { se mantiene como siempre }\end{array}$} & Mucho menos & Intermedio & Igual o más \\
\hline & & $158(24,4)[21,2-28,0]$ & $257(39,7)[36,0-43,6]$ & $231(35,7)[32,0-39,6]$ \\
\hline \multirow{2}{*}{5} & \multirow{2}{*}{$\begin{array}{l}\text { No consigo dormir } \\
\text { las horas necesarias }\end{array}$} & Nunca me ocurre & Intermedio & Constantemente \\
\hline & & $247(38,2)[34,4-42,1]$ & $357(55,2)[51,3-59,1]$ & $42(6,5)[4,7-8,7]$ \\
\hline \multirow{2}{*}{6} & \multirow{2}{*}{$\begin{array}{l}\text { Todo me aburre, incluso las cosas que } \\
\text { antes me divertían }\end{array}$} & No es cierto & Intermedio & Cierto \\
\hline & & $416(64,4)[60,5-68,0]$ & $193(29,8)[26,4-33,6]$ & $37(5,7)[4,1-7,8]$ \\
\hline \multirow{2}{*}{7} & \multirow{2}{*}{$\begin{array}{l}\text { Noto hormigueos en las manos y/o } \\
\text { los pies }\end{array}$} & No, en absoluto & Intermedio & Insoportable \\
\hline & & $363(56,1)[52,2-60,0]$ & $250(38,7)[34,9-42,5]$ & $33(5,1)[3,6-7,1]$ \\
\hline \multirow{2}{*}{8} & \multirow{2}{*}{$\begin{array}{l}\text { Me considero feliz en } \\
\text { mi relación de pareja }\end{array}$} & Nada & Intermedio & Completamente \\
\hline & & $107(16,5)[13,8-19,7]$ & $234(36,2)[32,5-40,0]$ & $305(47,2)[43,3-51,1]$ \\
\hline \multirow{2}{*}{9} & \multirow{2}{*}{$\begin{array}{l}\text { De pronto noto que empiezo a sudar } \\
\text { sin que haya hecho ningún esfuerzo }\end{array}$} & Nunca & Intermedio & Constantemente \\
\hline & & $286(44,2)[40,4-48,2]$ & $279(43,1)[39,3-47,1]$ & $81(12,5)[10,1-15,4]$ \\
\hline \multirow{2}{*}{10} & \multirow{2}{*}{ He perdido la capacidad de relajarme } & No, en absoluto & Intermedio & Completamente \\
\hline & & $403(62,3)[58,5-66,1]$ & $225(34,8)[31,1-38,6]$ & $18(2,7)[1,7-4,4]$ \\
\hline \multirow{2}{*}{11} & \multirow{2}{*}{$\begin{array}{l}\text { Aunque duermo, no consigo descan- } \\
\text { sar }\end{array}$} & Nunca me ocurre & Intermedio & Constantemente \\
\hline & & $284(43,9)[40,1-47,8]$ & $313(48,4)[44,5-52,3]$ & $49(7,5)[5,7-9,9]$ \\
\hline \multirow{2}{*}{12} & \multirow{2}{*}{$\begin{array}{l}\text { Noto como si las cosas } \\
\text { me dieran vueltas }\end{array}$} & Nada & Intermedio & Mucho \\
\hline & & $438(67,8)[64,0-71,3]$ & $195(30,1)[26,7-33,9]$ & $13(2,0)[1,1-3,5]$ \\
\hline \multirow{2}{*}{13} & Minonolsomencnocronoroiz & Nada importante & Intermedio & Muy importante \\
\hline & MI papel como esposa o pareja es... & $87(13,4)[10,9-16,4]$ & $153(23,6)[20,4-27,1]$ & $406(62,8)[58,9-66,5]$ \\
\hline 11 & Creo que retengo líquido, & No, como siempre & Intermedio & Sí, mucho más \\
\hline 14 & porque estoy hinchada & $464(71,8)[68,1-75,2]$ & $134(20,7)[17,7-24,1]$ & $48(7,4)[5,5-9,8]$ \\
\hline 15 & Estoy satisfecha con & Nada & Intermedio & Completamente \\
\hline 10 & mis relaciones sexuales & $180(2,9)[24,5-31,5]$ & $158(24,5)[21,2-28,0]$ & $307(47,6)[43,6-51,5]$ \\
\hline 16 & Noto que los músculos o & No, en absoluto & Intermedio & Dolor insoportable \\
\hline 10 & las articulaciones me duelen & $186(28,7)[25,3-32,4]$ & $420(65,0)[61,1-68,6]$ & $40(6,1)[4,5-8,4]$ \\
\hline 17 & Creo que los demás & No, en absoluto & Intermedio & Cierto \\
\hline 17 & estarían mejor sin mí & $557(86,2)[83,2-88,7]$ & $77(11,9)[9,5-14,7]$ & $12(1,8)[1,0-3,3]$ \\
\hline 18 & Me da miedo hacer esfuerzos & No, en absoluto & Intermedio & Mucho \\
\hline 18 & porque se me escapa la orina & $514(79,5)[76,2-82,5]$ & $117(18,1)[61,1-68,6]$ & $15(2,3)[4,5-84]$ \\
\hline 10 & Desde que me levanto & Nada & Intermedio & Mucho \\
\hline 19 & me encuentro cansada & $325(50,3)[46,3-54,2]$ & $282(43,6)[39,8-47,5]$ & $39(6,0)[4,3-8,2]$ \\
\hline 20 & Tengo tan buena salud como & No, en absoluto & Intermedio & Igual o mejor \\
\hline 20 & cualquier persona a mi edad & $86(13,3)[10,8-16,2]$ & $302(46,7)[42,8-50,6]$ & $258(39,9)[36,1-43,8]$ \\
\hline 21 & Tengo la sensación de que & Nunca & Intermedio & En todo momento \\
\hline 21 & no sirvo para nada & $544(84,2)[81,1-86,8]$ & $89(13,7)[11,2-16,7]$ & $13(2,0)[1,1-3,5]$ \\
\hline
\end{tabular}


Tabla 2. Manifestaciones menopáusicas identificadas con escala cervantes. Presencia y severidad $n=646$ (Continuación)

\begin{tabular}{|c|c|c|c|c|}
\hline \multirow{2}{*}{22} & \multirow{2}{*}{$\begin{array}{l}\text { Tengo relaciones sexuales } \\
\text { tan a menudo como antes }\end{array}$} & Mucho menos & Intermedio & Igual o más \\
\hline & & $270(41,8)[37,9-45,7]$ & $230(35,6)[31,9-39,4]$ & $146(22,6)[19,4-26,0]$ \\
\hline \multirow{2}{*}{23} & \multirow{2}{*}{$\begin{array}{l}\text { Noto que el corazón me late muy } \\
\text { deprisa y sin control }\end{array}$} & Nada & Intermedio & Mucho \\
\hline & & $462(71,5)[67,8-74,9]$ & $177(27,4)[24,0-31]$, & $7(1,0)[0,4-2,3]$ \\
\hline \multirow{2}{*}{24} & \multirow{2}{*}{$\begin{array}{l}\text { A veces pienso que no } \\
\text { me importaría estar muerta }\end{array}$} & Nunca & Intermedio & Constantemente \\
\hline & & $563(87,1)[84,2-89,5]$ & $76(11,7)[9,4-14,5]$ & $7(1,0)[0,4-2,3]$ \\
\hline \multirow{2}{*}{25} & \multirow{2}{*}{$\begin{array}{l}\text { Mi salud me causa problemas } \\
\text { con los trabajos domésticos }\end{array}$} & En absoluto & Intermedio & Constantemente \\
\hline & & $372(57,5)[53,6-61,4]$ & $249(38,5)[34,7-42,4]$ & $25(3,8)[2,5-5,7]$ \\
\hline \multirow{2}{*}{26} & \multirow{2}{*}{$\begin{array}{l}\text { En mi relación de pareja } \\
\text { me siento tratada de igual a igual }\end{array}$} & Nunca & Intermedio & Siempre \\
\hline & & $191(29,5)[26,1-33,2]$ & $127(19,6)[16,7-22,9]$ & $328(50,7)[46,8-54,6]$ \\
\hline \multirow{2}{*}{27} & \multirow{2}{*}{$\begin{array}{l}\text { Siento picor en la vagina, como si } \\
\text { estuviera demasiado seca }\end{array}$} & Nada & Intermedio & Mucho \\
\hline & & $483(74,7)[71,2-78,0]$ & $139(21,5)[18,4-24,9]$ & $24(3,7)[2,4-5,5]$ \\
\hline \multirow{2}{*}{28} & \multirow{2}{*}{ Me siento vacía } & Nunca & Intermedio & Siempre \\
\hline & & $511(79,1)[75,7-82,1]$ & $114(17,6)[14,8-20,8]$ & $21(3,2)[2,0-5,0]$ \\
\hline \multirow{2}{*}{29} & \multirow{2}{*}{ Noto sofocaciones } & Nunca & Intermedio & En todo momento \\
\hline & & $174(26,9)[23,5-30,5]$ & $351(54,3)[50,4-58,2]$ & $121(18,7)[15,8-22,0]$ \\
\hline \multirow[t]{2}{*}{30} & \multirow[t]{2}{*}{ En mi vida el sexo es } & Nada importante & Intermedio & $\begin{array}{l}\text { Extremadamente } \\
\text { importante }\end{array}$ \\
\hline & & $93(14,4)[11,8-17,4]$ & $188(29,1)[25,6-32,8]$ & $365(56,5)[52,5-60,3]$ \\
\hline \multirow{2}{*}{31} & \multirow{2}{*}{$\begin{array}{l}\text { He notado que tengo } \\
\text { más sequedad de piel }\end{array}$} & No, como siempre & Intermedio & Sí, mucho más \\
\hline & & $321(49,6)[45,7-53,6]$ & $254(39,3)[35,5-43,2]$ & $71(10,9)[8,7-13,7]$ \\
\hline
\end{tabular}

Tabla 3. Puntuación de cada manifestación menopáusica identificadas con escala Cervantes. Distribución según estado menstrual $n=646$

\begin{tabular}{|c|c|c|c|c|}
\hline & ITEM & $\begin{array}{l}\text { Premenopausia } \\
\qquad \mathrm{X} \pm \text { SD }\end{array}$ & $\begin{array}{l}\text { Posmenopausia } \\
\text { X } \pm \text { SD }\end{array}$ & $\mathbf{p}$ \\
\hline 1 & Durante el día noto que la cabeza me va doliendo cada vez más & $0,3 \pm 0,4$ & $0,5 \pm 0,5$ & $0,001^{*}$ \\
\hline 2 & No puedo más de lo nerviosa que estoy & $0,2 \pm 0,4$ & $0,3 \pm 0,4$ & $<0,001+$ \\
\hline 3 & Noto mucho calor de repente & $0,7 \pm 0,4$ & $0,8 \pm 0,3$ & $0,005+$ \\
\hline 4 & Mi interés por el sexo se mantiene como siempre & $0,8 \pm 0,3$ & $0,6 \pm 0,4$ & $<0,001+$ \\
\hline 5 & No consigo dormir las horas necesarias & $0,5 \pm 0,4$ & $0,7 \pm 0,4$ & $<0,001+$ \\
\hline 6 & Todo me aburre, incluso las cosas que antes me divertían & $0,2 \pm 0,4$ & $0,4 \pm 0,5$ & $<0,001^{*}$ \\
\hline 7 & Noto hormigueos en las manos y/o los pies & $0,3 \pm 0,4$ & $0,5 \pm 0,4$ & $<0,001^{*}$ \\
\hline 8 & Me considero feliz en mi relación de pareja & $0,8 \pm 0,3$ & $0,7 \pm 0,4$ & $<0,001+$ \\
\hline 9 & $\begin{array}{l}\text { De pronto noto que empiezo a sudar sin que haya hecho nin- } \\
\text { gún esfuerzo }\end{array}$ & $0,4 \pm 0,5$ & $0,6 \pm 0,4$ & $<0,001^{*}$ \\
\hline 10 & He perdido la capacidad de relajarme & $0,3 \pm 0,4$ & $0,4 \pm 0,4$ & $0,003^{*}$ \\
\hline 11 & Aunque duermo, no consigo descansar & $0,5 \pm 0,5$ & $0,6 \pm 0,4$ & $0,003^{*}$ \\
\hline 12 & Noto como si las cosas me dieran vueltas & $0,2 \pm 0,4$ & $0,4 \pm 0,4$ & $<0,001+$ \\
\hline 13 & Mi papel como esposa o pareja es importante & $0,8 \pm 0,3$ & $0,8 \pm 0,3$ & $0,006+$ \\
\hline 14 & Creo que retengo líquido, porque estoy hinchada & $0,1 \pm 0,3$ & $0,4 \pm 0,4$ & $<0,001+$ \\
\hline
\end{tabular}


Tabla 3. Puntuación de cada manifestación menopáusica identificadas con escala Cervantes. Distribución según estado menstrual $n=646$ (Continuación)

\begin{tabular}{|c|c|c|c|c|}
\hline 15 & Estoy satisfecha con mis relaciones sexuales & $0,7 \pm 0,4$ & $0,6 \pm 0,4$ & $0,074^{*}$ \\
\hline 16 & Noto que los músculos o las articulaciones me duelen & $0,6 \pm 0,4$ & $0,8 \pm 0,3$ & $<0,001+$ \\
\hline 17 & Creo que los demás estarían mejor sin mí & $0,1 \pm 0,3$ & $0,1 \pm 0,3$ & $0,011+$ \\
\hline 18 & Me da miedo hacer esfuerzos porque se me escapa la orina & $0,1 \pm 0,3$ & $0,2 \pm 0,4$ & $0,002+$ \\
\hline 19 & Desde que me levanto me encuentro cansada & $0,4 \pm 0,4$ & $0,6 \pm 0,4$ & $<0,001^{*}$ \\
\hline 20 & Tengo tan buena salud como cualquier persona a mi edad & $0,8 \pm 0,3$ & $0,8 \pm 0,3$ & $0,008+$ \\
\hline 21 & Tengo la sensación de que no sirvo para nada & $0,1 \pm 0,3$ & $0,2 \pm 0,4$ & $0,002+$ \\
\hline 22 & Tengo relaciones sexuales tan a menudo como antes & $0,6 \pm 0,4$ & $0,4 \pm 0,5$ & $<0,001^{*}$ \\
\hline 23 & Noto que el corazón me late muy deprisa y sin control & $0,2 \pm 0,4$ & $0,3 \pm 0,4$ & $<0,001+$ \\
\hline 24 & A veces pienso que no me importaría estar muerta & $0,0 \pm 0,2$ & $0,1 \pm 0,3$ & $0,006+$ \\
\hline 25 & Mi salud me causa problemas con los trabajos domésticos & $0,3 \pm 0,4$ & $0,5 \pm 0,4$ & $<0,001^{*}$ \\
\hline 26 & En mi relación de pareja me siento tratada de igual a igual & $0,7 \pm 0,4$ & $0,6 \pm 0,4$ & $0,331^{*}$ \\
\hline 27 & Siento picor en la vagina, como si estuviera demasiado seca & $0,1 \pm 0,3$ & $0,3 \pm 0,4$ & $<0,001+$ \\
\hline 28 & Me siento vacía & $0,1 \pm 0,3$ & $0,2 \pm 0,4$ & $0,002^{*}$ \\
\hline 29 & Noto sofocaciones & $0,7 \pm 0,4$ & $0,7 \pm 0,4$ & $0,056^{*}$ \\
\hline 30 & En mi vida el sexo es importante & $0,8 \pm 0,3$ & $0,7 \pm 0,4$ & $<0,001+$ \\
\hline 31 & He notado que tengo más sequedad de piel & $0,4 \pm 0,4$ & $0,5 \pm 0,4$ & $0,005+$ \\
\hline
\end{tabular}

$\left(^{*}\right)$ Anova. (+) Mann-Whitney

Tabla 4. Puntuación de dominios, subdominios y global de la escala cervantes. Distribución según estado menstrual

\begin{tabular}{|c|c|c|c|c|}
\hline & $\begin{array}{c}\text { Todas } \\
\mathrm{n}=646(100,0 \%)\end{array}$ & $\begin{array}{c}\text { Premenopausia } \\
\begin{array}{c}n=384 \\
(59,4 \%)\end{array}\end{array}$ & $\begin{array}{c}\text { Posmenopausia } \\
\begin{array}{c}n=262 \\
(40,6 \%)\end{array}\end{array}$ & $\mathbf{P}$ \\
\hline & & $\mathrm{X} \pm \mathrm{DE}$ & & \\
\hline Menopausia y salud & $20,2 \pm 12,3$ & $17,7 \pm 11,9$ & $23,8 \pm 11,8$ & $<0,001$ * \\
\hline Psíquico & $5,9 \pm 7,1$ & $4,7 \pm 6,3$ & $7,7 \pm 7,9$ & $<0,001+$ \\
\hline Sexualidad & $10,5 \pm 4,9$ & $9,3 \pm 4,7$ & $12,2 \pm 4,8$ & $<0,001 *$ \\
\hline Relación de pareja & $4,5 \pm 5,1$ & $3,9 \pm 4,7$ & $5,6 \pm 5,5$ & $<0,001+$ \\
\hline Síntoma vasomotor & $6,4 \pm 4,8$ & $6,0 \pm 5,0$ & $7,0 \pm 4,4$ & $0,002+$ \\
\hline Salud & $5,5 \pm 4,7$ & $4,9 \pm 4,4$ & $7,1 \pm 4,8$ & $<0,001+$ \\
\hline Envejecimiento & $8,2 \pm 5,9$ & $7,0 \pm 5,6$ & $10,1 \pm 5,8$ & $<0,001$ * \\
\hline Puntaje global & $39,2 \pm 22,6$ & $33,2 \pm 20,4$ & $48,0 \pm 22,7$ & $<0,001$ * \\
\hline
\end{tabular}

$\left({ }^{*}\right)$ Anova. (+) Mann-Whitney 
Tabla 5. Factores asociados a deterioro de la calidad de vida. Puntuación sobre la media. Escala Cervantes $n=646$

\begin{tabular}{|c|c|c|c|}
\hline & $\mathrm{X} \pm \mathrm{SD}$ & OR & $\mathbf{P}$ \\
\hline \multicolumn{4}{|c|}{ Grupos etarios } \\
\hline $40-44$ & $27,8 \pm 19,9$ & 1 & 1 \\
\hline $45-49$ & $38,3 \pm 21,4$ & $6,59[4,13-10,51]$ & $<0,001$ \\
\hline $50-54$ & $45,6 \pm 22,3$ & $9,80[5,93-16,20]$ & $<0,001$ \\
\hline $55-59$ & $47,7 \pm 21,7$ & $11,32[6,87-18,65]$ & $<0,001$ \\
\hline \multicolumn{4}{|c|}{ Estado nutricional } \\
\hline Normal & $36,9 \pm 23,4$ & 1 & 1 \\
\hline Infrapeso & $3,6 \pm 24,8$ & $2,95[1,01-8,65]$ & 0,04 \\
\hline Preobesidad & $39,1 \pm 22,5$ & $2,70[1,84-3,95]$ & $<0,001$ \\
\hline Obesidad I & $40,8 \pm 22,6$ & $3,20[1,98-5,16]$ & $<0,001$ \\
\hline Obesidad II & $45,1 \pm 17,0$ & $4,86[2,48-9,56]$ & $<0,001$ \\
\hline Obesidad III & $44,6 \pm 26,4$ & $4,42[1,07-18,26]$ & 0,04 \\
\hline \multicolumn{4}{|c|}{ Nivel de escolaridad } \\
\hline Profesional & $26,5 \pm 19,2$ & 1 & 1 \\
\hline Bachiller & $42,9 \pm 24,1$ & $9,82[5,50-14,29]$ & $<0,001$ \\
\hline Técnico & $46,6 \pm 25,8$ & $6,88[5,99-16,12]$ & $<0,001$ \\
\hline Primaria & $42,3 \pm 20,6$ & $8,87[2,44-19,36]$ & $<0,001$ \\
\hline \multicolumn{4}{|c|}{ Años de formación académica } \\
\hline 12 años o más & $29,5 \pm 21,0$ & 1 & 1 \\
\hline Menos de 12 años & $42,5 \pm 22,1$ & $8,65[0,76-1,48]$ & $<0,001$ \\
\hline \multicolumn{4}{|c|}{ Habido de fumar } \\
\hline Nunca & $36,0 \pm 20,9$ & 1 & 1 \\
\hline Anteriormente & $49,1 \pm 23,0$ & $9,99[5,91-16,89]$ & $<0,001$ \\
\hline Actualmente & $55,4 \pm 25,4$ & $17,42[9,45-32,10]$ & $<0,001$ \\
\hline \multicolumn{4}{|c|}{ Uso actual de terapia hormonal } \\
\hline Si & $46,3 \pm 27,4$ & 1 & 1 \\
\hline No & $38,8 \pm 22,3$ & $3,92[1,90-8,09]$ & $<0,001$ \\
\hline \multicolumn{4}{|c|}{ Estado menopaúsico } \\
\hline Premenopausia & $48,0 \pm 22,7$ & 1 & 1 \\
\hline Posmenopausia & $37,5 \pm 22,2$ & $9,27[6,43-13,36]$ & $<0,001$ \\
\hline \multicolumn{4}{|c|}{ Clasificación de las etapas en la postmenopausia } \\
\hline Postmenopausia temprana & $46,8 \pm 22,0$ & 1 & 1 \\
\hline Postmenopausia tardía & $45,3 \pm 21,8$ & $1,98[1,26-3,11]$ & 0,003 \\
\hline Postmenopausia alejada & $59,3 \pm 26,6$ & $5,68[2,75-11,74]$ & $<0,001$ \\
\hline
\end{tabular}

\section{DISCUSIÓN}

Varias escalas han sido propuestas para evaluar CV en el climaterio, siendo traducidas y validadas al español $(1-4,15)$. La única creada en esta lengua y específica para menopausia es EC (5).

Oleadas de calor es la manifestación más ampliamente considerada del climaterio, EC permite explorar dicha manifestación por medio de tres preguntas, valora el mismo síntoma desde tres percepciones distintas de la mujer: "notar mucho calor de repente", "notar sofocaciones" $y$ "de pronto notar que empieza a sudar sin que haya hecho ningún esfuerzo". Entre las afrocolombianas estudiadas se encontró que el $76,1 \% ; 73,1 \%$ y $55,8 \%$ respectivamente las presentaban, no se logró identificar otros estudios con EC en otras comunidades afrodescendientes o africanas. Con la versión corta, EC-16 (16) estudiaron 317 mujeres 
españolas con edad 55,7 $\pm 5,3$ años, sin que precisaran aspectos étnicos, encontrando oleadas de calor en $73,8 \%$. En 300 mujeres ecuatorianas, edad media 45 años y poca presencia de afrodescendientes, el 65,5\% informaron oleadas de calor cuando se valoraron con Menopause Rating Scale (MRS), herramienta diferente a EC (15).

Entre las afrocolombianas posmenopáusicas se encontró siognificativamente mayor puntuación media en "notar mucho calor de repente" $y$ "sudar sin haber hecho ningún esfuerzo" que en las premenopáusicas, iơual a lo señalado por otros autores (17). Las posmenopáusicas, sobre todo tempranas, son más propensas a tener oleadas de calor en relación con la reducción en los niveles o picos de estrógeno, las modificaciones en la liberación de las gonadotropinas, así como con su interrelación con el centro termorregulador cerebral (11). Los mismos mecanismos explican las oleadas de calor de la transición a la menopausia o premenopausia, ya que dicho síntoma suele tener presencia en mujeres que aún menstrúan y están en etapa de climaterio $(10,12)$.

Con datos del proyecto CAVIMEC, al parecer por vez primera se señaló la importancia del dolor musculo/ articular en afrodescendientes en climaterio, al encontrar con escala MRS que era la primera manifestación menopaúsica, con prevalencia del 80\% (18). En el presente estudio, con EC se encontró que el 71,3\% la manifestaban, siendo sionificativamente mayor la puntuación media entre las que no menstruaban, indicando que las posmenopáusicas tenían más dolor musculo/articular que las premenopáusicas. Monterrosa (19) estudió 1.215 mujeres con MRS, incluyendo afrodescendientes, indígenas e hispánicas caribeñas colombianas y encontró que el 72,9\% informaron molestias músculo/articular, siendo el síntoma menopaúsico más frecuente e identificaron que la tercera parte señaló que era severa/muy severa dicha manifestación. EC también permite valorar la severidad (5), en el presente estudio 40 mujeres informaron dolor musculo/articular insoportable y 420 tenía dolor musculo/articular en diferente magnitud. Desde la creación de la EC se han intentado versiones cortas $(14,20)$, una de ellas con 10 preguntas estudió 1.739 colombianas, incluyendo 30,9\% afrodescendientes y también encontró que la manifestación más prevalente fueron los dolores musculo/articulares: $71,2 \%$, teniendo el 20,6\% importante severidad (20). En 2.218 mujeres, estudiadas de la base de datos SWAN que incluía $27,0 \%$ afroamericanas, se encontró elevada prevalencia de dolores musculo/articulares, una de cada seis reportó que los síntomas eran diarios y las posmenopáusicas significativamente tenían mayor presencia que las premenopáusicas (21). La elevada presencia de dolor musculo/articular se explica por modificaciones en neurotransmisores, osteoartritis, fibromialgia y cambios hormonales (19).

Otra importante manifestación en el climaterio son los disturbios del dormir $(8,9)$. Si bien existen herramientas específicas $(22,23)$, EC permite aproximación con tres preguntas (5). Se encontró que el $61,8 \%$ informaron no conseguir dormir las horas necesarias, $56,1 \%$ que aunque dormían no descansaban y 49,7\% desde la levantada estaban cansadas. Cifras similares a las señaladas por otros autores con otras herramientas, lo que permite señalar que la mitad de las mujeres climatéricas tienen percepción de mal dormir, condicionante para deterioro de la $C V$ así como factor de riesgo asociado a algunas enfermedades crónicas, especialmente cardiovascular con impacto en la morbilidad e incluso mortalidad (22-24). En un estudio a gran escala en 1.078 mujeres colombianas, edad 40-59 años, siendo 86,1\% mestizas y 13,9\% afrocolombianas, valoradas con el Índice de Calidad deI Sueño de Pittsburgh, se estimó prevalencia de mala calidad del sueño del 57,1\% (24).

Son diversas las manifestaciones que permite explorar EC, algunas con mayor o menor impacto según las prioridades de atención en salud, pero todas de importancia desde la perspectiva de la mujer (5), permitiendo visibilizar síntomas poco tenidos en cuenta, por las limitaciones para explicar su relación con las modificaciones en los niveles del estradiol $(6,25)$. Por ejemplo, la mitad de las afrocolombianas estudiadas presentaban cefalea que se iba incrementando a medida que pasaba el día y el 2,0\% informaron que se presentaba todos los días. Se identifica una manifestación que deteriora la $C \nabla$ y puede repercutir adversamente en la productividad personal, familiar e incluso social, con detrimento del desempeño en la actividad física cotidiana o en el rol comunitario. Se debe cuantificar la magnitud del impacto de todas las manifestaciones en la actividad física diaria, como realizó Gibson (17) con las oleadas de calor. 
No solo síntomas biológicos se deben valorar, manifestaciones psicológicas, sexuales y sociales deben ser de interés $(3,4)$. EC explora manifestaciones psicológicas con 9 preguntas y las agrupa en el dominio psíquico (5). La tercera parte de la afrocolombianas valoradas tenía síntomas psicológicos, se ha señalado previamente que afrocolombianas climatéricas tienen mayor deterioro psicológico que mestizas o indígenas (18). No se conocen explicaciones para esas diferencias. La pérdida acelerada de folículos ováricos y el hipoestrogénismo, conllevan reducción en la biodisponibilidad de neurotransmisores: adrenalina, acetilcolina, histamina, glutamato, aspartato, glicina, $\beta$-alanina y taurina. Los cambios neuroendocrinos en distintas áreas del cerebro, ocasionan sintomatología vasomotora, trastornos en la conducta alimentaria y mayor presencia de depresión, ansiedad e irritabilidad (25). En el estudio las afrocolombianas posmenopáusicas tuvieron peor puntuación en el dominio psíquico, que las premenopáusicas.

La EC con 4 preguntas aborda la sexualidad, aunque carece del dimensionamiento para establecer disfunción sexual (5). Entre las afrocolombianas estudiadas: $15 \%$ señaló que el sexo era nada importante, $56 \%$ que era extremadamente importante, $41 \%$ tenía relaciones sexuales mucho menos que antes $y$ para el $24 \%$ el interés por el sexo era mucho menor. Un estudio que hace parte de CAVIMEC, realizado en 461 afrocolombianas con edad 47,6 $\pm 5,4$ años, evaluadas con el Índice de Función Sexual Femenina, encontró que el $66,2 \%$ eran sexualmente activas y el dominio más deteriorado fue deseo sexual, seguido de dolor coital. La prevalencia de disfunción sexual fue: $38.4 \%$, siendo hábito de fumar, OR: 3.3 [IC95\%: 1,0-10.6; $\mathrm{p}=0,041$ ], hipertensión arterial, OR: 2.2 [IC95\%: 1.14.4; $\mathrm{p}=0.026]$ y escolaridad mayor de diez años, $O R$ : 0.4 [IC95\%: 0.2-0.8; $\mathrm{p}=0.003$ ], factores asociados a disfunción sexual. (26).

REDLINC evaluó disfunción sexualy factores asociados en latinoamericanas de mediana edad, también con el Índice de Función Sexual Femenina (27), estudio multicéntrico estando el $41 \%$ en posmenopausia. La prevalencia de disfunción sexual fue del $56,8 \%$, siendo diferente según los países, desde el 21,0\% (Cartagena) hasta 98,5\% (Quito). Las cartageneras participantes eran afrocolombianas, observándose prevalencias similares e igualmente bajas en las cubanas, dominicanas y panameñas, países donde la presencia de comunidades afrodescendientes es importante. En los países con elevada presencia de indígenas como Ecuador, Bolivia y Perú, fue alta la presencia de disfunción sexual. Las afrocolombianas tienen menor deterioro urogenital que mestizas o indígenas cuando son valoradas con una escala diferente a la EC (18). Factores nutricionales, hábitos, genéticos, patrones culturales, educativos, tradiciones o sociales, influyen $y$ generan diferencias cuando se estudia disfunción sexual (26). La sexualidad involucra el estado saludable de la mucosa vaginal, apreciaciones psíquicas, ambientales, personales y las propias del compañero sexual (27).

La EC (5) permite precisar aspectos referentes específicamente al envejecimiento y relación de pareja, siendo valiosa cualidad de la herramienta. La relación de pareja la aborda por medio de tres preguntas. El 16,5\% de las mujeres estudiadas se consideraban nada feliz con su relación de pareja, el 13,4\% consideraron nada importante su papel como esposa o pareja y sólo la mitad se sentían tratadas de igual a igual por su pareja. Si bien estas manifestaciones pudiesen no guardar relación con los aspectos biológicos de la pérdida de la actividad ovárica, son condicionantes importantes para el deterioro de la CV. Situaciones sociales relevantes como la violencia doméstica, la discriminación social y étnica, así como el imaginario social en el cual se ubica a la mujer climatérica, impactan adversamente a la mujer. Se encontró peor relación de pareja en las posmenopáusicas con respecto a las que aún presentaban menstruaciones.

EC al poseer subdominios $y$ dominios permite explorar holísticamente los estados menopaúsicos en particular y el climaterio en general, para identificar aspectos del deterioro de la $C \nabla$, más allá de solo la presencia de síntomas (6). Entre las afrocolombianas estudiadas que aún menstruaban, la puntuación del dominio menopausia y salud fue 17,7 $\pm 11,9$, mientras que entre las que habían dejado de menstruar fue $23,8 \pm 11,8$, lo que permite señalar que las ultimas tiene mayor deterioro que las primeras, por tanto en ellas debiesen generarse mayores acciones de atención en salud.

En un editorial del año 2017 Styer (28) señaló la importancia del abordaje de las manifestaciones, patologías y respuesta a medicación teniendo en 
cuenta etnia/raza, ya que existen diferencias en cuanto a enfermedad cardiovascular, hipertensión arterial, diabetes y en el beneficio de los estrógenos en mujeres posmenopáusicas. EI proyecto CAVIMEC $(7,8,18,19)$ con escala MRS encontró mayor presencia de síntomas urogenitales y disfunción sexual en indígenas colombianas en climaterio que en afrodescendientes, estas presentaban más dolor musculo/articular que mestizas y en las últimas las oleadas de calor eran más prevalentes que en las otras dos etnias. Hábitos alimenticios, mitos, estereotipos sociales, pautas comportamentales y genéticos puedes estar involucrados (9). La disponibilidad de tecnología para estudiar el porcentaje de presencia de genes de ancestría, es valiosa herramienta para estudiar a las mujeres en climaterio de acuerdo a su etnia/raza (28).

El estudio tiene como limitaciones las propias de los estudios transversales $y$ pese a ser realizado en un amplio grupo de afrocolombianas, no se adelantó con un muestreo específico poblacional. Se consideró la autodeterminación étnica de las personas y para otros estudios dentro de la misma línea debiese considerarse las prevalencias de genes de ancestría. Sus resultados no deben necesariamente ser extrapolados a todos los conglomerados de afrocolombianas. Hacen falta estudios en otras comunidades afrodescendientes latinoamericanas para hacer mayores precisiones. Tiene como fortaleza ser el primer estudio con EC en afrodescendientes $y$ haberse adelantado en población general y no en pacientes de consulta médica. Visibiliza manifestaciones que expresaron tener afrocolombianas en climaterio $y$ permite observar el comportamiento según estados menstruales. Se debe mirar como aporte inicial para abordar a la mujer afrocolombiana, que tiene la necesidad de ser manejada integralmente en el climaterio. El abordaje de las manifestaciones que deterioran la $C V$ debe ser elemento de la atención primaria de las climatéricas, sea realizado por médicos o enfermeras.

La menopausia se debe desmitificar y no permitir que sea obligatoria y globalmente razón para medicar. La menopausia no debe ser herramienta para discriminar. Se recomienda a los entes gubernamentales, a las empresas e instituciones prestadoras de salud, como a todos los actores del sector que evalúan, atienden o asisten a mujeres etapa de climaterio, explorar desde sus sitiales, la presencia de síntomas que se han declarado directa o indirectamente relacionados con la menopausia, teniendo en cuenta un factor diferenciador, la etnia a la cual pertenece la mujer.

\section{CONCLUSIÓN}

En afrocolombianas en etapa de climaterio se observó importante presencia de manifestaciones menopaúsicas cuando se valoraron con EC. Siete de cada diez manifestaron oleadas de calor o dolor musculo/articular. Fue mayor el deterioro de la $C V$ en las posmenopáusicas.

\section{CONFLICTOS DE INTERESES}

Ninguno que declarar.

\section{FINANCIACIÓN}

Recursos financieros suministrados por la Universidad de Cartagena, Colombia. Plan de fortalecimiento a la investigación para Grupos categorizados por Colciencias y avalados por la Universidad de Cartagena. Resolución 03707 del 2014. Acta de compromiso 070-2015 entre la Universidad de Cartagena y el Grupo de Investigación Salud de la Mujer.

\section{REFERENCIAS BIBLIOGRÁFICAS}

1. The WHOQOL Group. The World Health Organization Quality of Life Assessment (WHOQOL): development and general psychometric properties. Soc Sci Med. 1998 Jun;46(12):1569-85. DOI 10.1016/S02779536(98)00009-4.

2. Hilditch JR, Lewis J, Peter A, van Maris B, Ross A, Franssen E, et al. A menopause-specific quality of life questionnaire: development and psychometric properties. Maturitas. 1996 Jul;24(3):161-75. Erratum in: Maturitas 1996 Nov;25(3):231.

3. Greene JG. Constructing a standard climacteric scale. Maturitas. 1998 May;29(1):25-31.

4. Berlin Center for Epidemiology and Health Research [Internet]. Berlin: MSR; 2008 [cited 2011 Sept 25]. MRS. The Menopause Rating Scale. Available from: http://www.menopause-rating-scale.info/about.htm 
5. Palacios S, Ferrer-Barriendos J, Parrilla JJ, CasteloBranco C, Manubens M, Alberich X, et al. [Healthrelated quality of life in the Spanish women through and beyond menopause. Development and validation of the Cervantes Scale]. Med Clin (Barc). 2004 Feb;122(6):205-11. Spanish.

6. Avis NE, Stellato R, Crawford S, Bromberger J, Ganz p Cain $\nabla$, et al. Is there a menopausal syndrome? Menopausal status and symptoms across racial/ethnic groups. Soc Sci Med. 2001 Feb;52(3):345-56.

7. Monterrosa A, Blumel JE, Chedraui P, Gomez B, Valdez C. Quality of life impairment among postmenopausal women varies according to race. Gynecol Endocrinol. 2009 Aug;25(8):491-7.

8. Monterrosa-Castro A. Calidad de vida en la menopausia y etnias colombianas. Primeros resultados del estudio CAVIMEC. Rev Colomb Menopaus. 2011;17(4):70-81.

9. Romero-Pérez IM, Monterrosa-Castro A, PaterninaCaicedo A. Menopausia y etnias/razas: ¿hay diferencias en la presentación de los síntomas? Rev Colomb Obstet Ginecol. 2010;61(4):319-328.

10. Miller SR, Gallicchio LM, Lewis LM, Babus JK, Langenberg $\mathrm{P}$, Zacur HA, et al. Association between race and hot flashes in midlife women. Maturitas. 2006 Jun;54(3):260-9.

11. Freeman EW, Grisso JA, Berlin J, Sammel M, GarciaEspana B, Hollander L. Symptom reports from a cohort of African American and white women in the late reproductive years. Menopause. 2001 JanFeb;8(1):33-42.

12. Gold EB, Sternfeld B, KeIsey JL, Brown C, Mouton C, Reame N, et al. Relation of demographic and lifestyle factors to symptoms in a multi-racial/ethnic population of women 40-55 years of age. Am J Epidemiol. 2000 Sep;152(5):463-73.

13. Departamento Administrativo Nacional de Estadísticas [Internet]. Bogotá: DANE [consultado 2016 Jul 23]. Estadísticas por tema: Demografía y Población. Disponible en: https://www.dane.gov.co/index.php/ estadisticas-por-tema/demografia-y-poblacion

14. Colombia. Ministerio de Salud. Resolución No 008430 DE 1993 por la cual se establecen las normas científicas, técnicas y administrativas para la investigación en salud. Bogotá: El ministerio; Octubre 4 de 1993. Disponible en: http://www.urosario.edu.co/urosario_files/a2/ a24fb07a-f561-4fcc-b611-affff4374bb7.pdf
15. Chedraui P, Aguirre W, Hidalgo L, Fayad L. Assessing menopausal symptoms among healthy middle aged women with the Menopause Rating Scale. Maturitas. 2007 Jul;57(3):271-8.

16. Coronado PJ, Sánchez-Borrego R, Ruiz MA, Baquedano L, Sánchez S, Argudo C, et al. Psychometric attributes of the Cervantes short-form questionnaire for measuring health-related quality of life in menopausal women. Maturitas. 2016 Feb;84:55-62. DOI 10.1016/j.maturitas.2015.10.013.

17. Gibson C, Matthews K, Thurston R. Daily physical activity and hot flashes in the Study of Women's Health Across the Nation (SWAN) Flashes Study. Fertil Steril. 2014 Apr;101(4):1110-6. DOI 10.1016/j.fertnstert.2013.12.029.

18. Monterrosa-Castro A, Blumel JE, Chedraui P. Frecuencia y severidad de síntomas menopáusicos en una población de mujeres afrodescendientes colombianas. MedUnab. 2008;11(2):113-119.

19. Monterrosa-Castro A, Romero-Pérez I, Paternina-Caicedo A. Manifestaciones músculo-articulares, y no las oleadas de calor, es el síntoma más prevalente en mujeres climatéricas del Caribe colombiano. Salud Uninorte. 2010;26(2):179-88.

20. Pérez-López FR, Fernández-Alonso AM, Pérez-Roncero G, Chedraui P, Monterrosa-Castro A, Llaneza P. Assessment of menopause-related symptoms in mid-aged women with the 10-item Cervantes Scale. Maturitas. 2013 Oct;76(2):151-4. DOI 10.1016/j.maturitas.2013.07.002.

21. Dugan SA, Powell LH, Kravitz HM, Everson Rose SA, Karavolos K, Luborsky J. Musculoskeletal pain and menopausal status. Clin J Pain. 2006 May;22(4):325-31.

22. Buysse DJ, Reynolds CF 3rd, Monk TH, Berman SR, Kupfer DJ. The Pittsburgh Sleep Quality Index: a new instrument for psychiatric practice and research. Psychiatry Res. 1989 May;28(2):193-213.

23. Soldatos CR, Dikeos DG, Paparrigopoulos TJ. The diagnostic validity of the Athens Insomnia Scale. J Psychosom Res. 2003 Sep;55(3):263-7.

24. Monterrosa-Castro A, Marrugo-Flórez M, RomeroPérez I, Fernández-Alonso AM, Chedraui P, PérezLópez FR. Assessment of sleep quality and correlates in a large cohort of Colombian women around menopause. Menopause. 2013 Apr;20(4):464-9. DOI 10.1097/gme.0b013e31826e7649.

25. Bocchino S. Aspectos psiconeuroendocrinos de la perimenopausia, menopausia y climaterio. Rev Psiquiatr Urug. 2005;70(1):66-79. 
26. Monterrosa-Castro A, Márquez-Vega J, Arteta-Acosta C. Disfunción sexual en mujeres climatéricas afrodescendientes del Caribe Colombiano. Iatreia. 2014 Ene-Mar; 27(1):31-41.

27. Blümel JE, Chedraui P, Baron G, Belzares E, Bencosme A, Calle A, et al. Sexual dysfunction in middle-aged women: a multicenter Latin American study using the Female Sexual Function Index.
Menopause. 2009 Nov-Dec;16(6):1139-48. DOI 10.1097/ gme.0b013e3181a4e317.

28. Styer AK. The impact of estrogen alone hormone therapy on breast cancer risk and health outcomes: reassurance for the treatment of climacteric symptoms in black women? Menopause. 2017 Feb;24(2):124-125. DOI 10.1097/GME.0000000000000821. 